weiß, dass eine mindestens 60-tägige Behandlung das Risiko für Zysten im ZNS reduziert, ist eine Therapie der asymptomatischen konnatalen Toxoplasmose für drei Monate als sinnvoll zu erachten.

Die unerwünschten Wirkungen der Therapie werden möglicherweise überschätzt: In einer Studie, in der eine Behandlung einer konnatalen Toxoplasmose mit Pyrimethamin und Sulfadoxin über einen Zeitraum von 12 Monaten bei 65 Kindern überprüft wurde, trat bei drei Kindern eine Neutropenie, bei zwei eine Eosinophilie und bei einem sowohl eine Anämie als auch eine Eosinophilie auf. Bei vier der Kinder wurde die Be- handlung zeitweise unterbrochen und in nur einem Fall wurde eine Toxizität beobachtet, nachdem die Therapie wieder aufgenommen wurde. Bei neun weiteren Kindern kam es zu milden unerwünschten Ereignissen wie Übelkeit und Erbrechen, die Medikation wurde nicht gestoppt [Teil J et al. Pediatr Infect Dis J 2016;35:634-8].

Da bei asymptomatischer konnataler Toxoplasmose mit zunehmender Lebensdauer - und damit Immunkompetenz - das therapeutische Fenster kleiner wird und weil ohne Therapie die Wahrscheinlichkeit steigt, dass sich neue Zysten entwickeln, sollte die Behandlung so früh wie möglich erfolgen. Solange die Ergebnisse der französischen Vergleichsstudie noch nicht vorliegen, sollte das konventionelle Therapieschema mit Pyrimethamin, Sulfadiazin und Folinsäure bevorzugt werden. Danach sollten die Therapieempfehlungen gegebenenfalls aktualisiert werden.

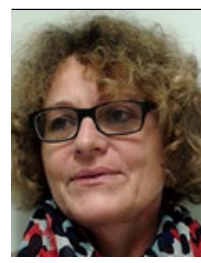

Dr. Ingrid Reiter-Owona Institut für Medizinische Mikrobiologie, Immunologie und Parasitologie Universitätsklinik Bonn Sigmund-Freud-Straße 25 53105 Bonn

\title{
Kontra: Therapieempfehlung revidieren!
}

W enn eine Therapie geplant wird, sollte überlegt werden, welches die Therapieziele sind, ob eine effektive Therapie bekannt ist, ob es für die Dauer der Therapie evidenzbasierte Daten gibt und ob die Therapie mehr nutzt als sie schadet. Mögliche Ziele bei der Therapie der asymptomatischen konnatalen Toxoplasmose können eine Eradikation der Erreger, eine Verhinderung neurologischer Komplikationen und eine Reduktion des Chorioretinitis-Risikos sein. Mit den bislang zur Verfügung stehenden Medikamenten ist jedoch eine Eradikation der Toxoplasmose nicht möglich, das heißt die Infektion bleibt lebenslang bestehen. Zur Verhinderung neurologischer Komplikationen der konnatalen Toxoplasmose gibt es keine Daten, die belegen, dass die Kinder im Vergleich zu einer unbehandelten Kontrollgruppe von einer Therapie profitieren. Es liegt lediglich eine Studie vor, in der die Folgen einer mütterlichen Primärinfektion mit Toxoplasma gondii während der Schwangerschaft bei Kindern im Alter von drei bis vier Jahren untersucht wurden [Freeman $\mathrm{K}$ et al. BMC Pediatr 2005;5:23]. Es zeigte sich, dass Beeinträchtigungen der neurologischen Entwicklung und abweichendes Verhalten bei Kindern mit behandelter konnataler Toxoplasmose $(\mathrm{n}=178)$ gleich häufig waren wie bei Kindern ohne konnatale Toxoplasmose $(\mathrm{n}=527)$.
In Bezug auf das Risiko einer Chorioretinitis ergab eine Studie, in der 477 Kinder mit Toxoplasmose im Median 10,5 Jahre (bis zu 22 Jahre) nachbeobachtet wurden, dass bei fast $30 \%$ der $\mathrm{Pa}$ tienten trotz Therapie mindestens eine okuläre Läsion auftrat, bei $80 \%$ von ihnen ohne Visusbeeinträchtigung. Das Durchschnittsalter bei Erstmanifestation lag bei 3 Jahren. Bei mehr als einem Drittel von ihnen kam es im Verlauf von 12 Jahren zu weiteren Läsionen [Wallon $\mathrm{M}$ et al. Pediatrics 2014;133: e601-8].

In einer weiteren prospektiven Kohortenstudie, in der 281 Kinder mit konnataler Toxoplasmose im Durchschnitt 4,1 Jahre nachbeobachtet wurden, entwickelten 50 eine Chorioretinitis, bei 17 von ihnen trat diese rezidivierend auf [Freeman Ket al. Pediatrics 2008;121: e1215-22]. Bei den $92 \%$ der Kinder, bei denen in den ersten vier Lebensmonaten keine Anzeichen einer Chorioretinitis gefunden wurden, lag das Risiko, bis zum Alter von vier Jahren eine solche zu entwickeln, bei $8 \%$. Den Autoren zufolge rechtfertigt bei Kindern, die im frühen Alter keine auffälligen Augenveränderungen aufweisen, dieses geringe Risiko nicht, eine postnatale Therapie durchzuführen.

Somit gibt es keinen gesicherten Nachweis, dass die postnatale Therapie der Toxoplasmose Einfluss auf das Auftreten von Augenläsionen hat, analog zur fehlenden Wirksamkeit der Antiparasitika auf Verlauf und Rezidivhäufigkeit der Toxoplasmose-Chorioretinitis im 3. und 4. Lebensjahrzehnt. Gleichzeitig würde eine Therapie bedeuten, das Risiko von Nebenwirkungen in Kauf zu nehmen. Für die Therapie mit Pyrimethamin und Sulfadiazin wurden die potenziell letalen Lyell-Syndrom und „drug reaction with eosinophilia and systemic symptoms“ (DRESS) beschrieben. Hinzu kommt, dass die Anwendung von Antibiotika insbesondere im ersten Lebensjahr ungünstige Auswirkungen auf das Mikrobiom des Darms haben kann. Die Aussage im RKI-Ratgeber für Ärzte, es sei Experten zufolge unzulässig, Neugeborenen mit gesicherter klinisch apparenter oder inapparenter Infektion eine Therapie zu verweigern, sollte daher revidiert werden: Bei der asymptomatischen konnatalen Toxoplasma sollte eine antiparasitäre Therapie nicht empfohlen werden. Die postpartale Therapie bei symptomatischer konnataler Toxoplasmose sollte ähnlich wie bei der Chorioretinitis bei älteren Patienten auf drei bis sechs Wochen beschränkt werden.

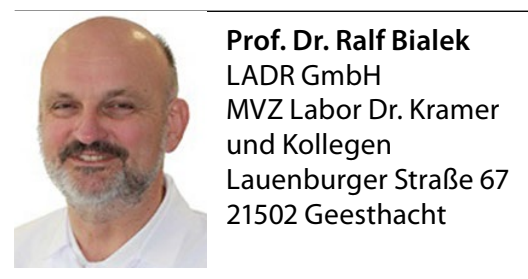

